Robotica: (2019) volume 37, pp. 2014-2034. (C) Cambridge University Press 2019. This is an Open Access article, distributed under the terms of the Creative Commons Attribution-NonCommercial-ShareAlike licence

(http://creativecommons.org/licenses/by-nc-sa/4.0/), which permits non-commercial re-use, distribution, and reproduction in any medium, provided the same Creative Commons licence is included and the original work is properly cited. The written permission of Cambridge University Press must be obtained for commercial re-use.

doi:10.1017/S0263574719000067

\title{
Design and Evaluation of a Soft Assistive Lower Limb Exoskeleton
}

\author{
Christian Di Natali $† *$, Tommaso Poliero $\dagger, \ddagger$ \\ Matteo Sposito†, Eveline Graf $\llbracket$, Christoph Bauer $\uparrow$, \\ Carole Pauli $\uparrow$, Eliza Bottenberg§, Adam De Eyto\|, \\ Leonard O’Sullivan\|, Andrés F. Hidalgo††, \\ Daniel Scherlył‡, Konrad S. Stadlerł‡, \\ Darwin G. Caldwell $†$ and Jesús Ortiz†®
}

\author{
$\dagger$ Department of Advanced Robotics, Istituto Italiano di Tecnologia, Genoa, Italy \\ E-mails:tommaso.poliero@iit.it,matteo.sposito@iit.it,darwin.caldwell@iit.it,jesus.ortiz@iit.it \\ $\ddagger$ Department of Informatics, Bioengineering, Robotics and Systems Engineering, University of \\ Genoa, Genoa, Italy \\ IInstitute of Physiotherapy, ZHAW Zurich University of Applied Sciences, Winterthur, Switzerland \\ E-mails:grav@zhaw.ch,bauc@zhaw.ch,carole.pauli@zhaw.ch \\ $\S$ Smart Functional Materials Research Group, Saxion University of Applied Sciences, Enschede, \\ The Netherlands. E-mail: e.bottenberg@saxion.nl \\ ||Design Factors Group, University of Limerick, Limerick, Ireland. E-mails: adam.deeyto@ul.ie, \\ leonard.osullivan@ul.ie \\ $\dagger \dagger$ Centre for Automation and Robotics Consejo Superior de Investigaciones Cientificas (CSIC), \\ Madrid, Spain.E-mail: af.hidalgo@csic.es \\ $\$$ Institute of Mechatronic Systems, ZHAW Zurich University of Applied Sciences, Winterthur, \\ Switzerland.E-mails: scey@zhaw.ch, stdl@zhaw.ch
}

(Accepted January 1, 2019. First published online: February 26, 2019)

\begin{abstract}
SUMMARY
Wearable devices are fast evolving to address mobility and autonomy needs of elderly people who would benefit from physical assistance. Recent developments in soft robotics provide important opportunities to develop soft exoskeletons (also called exosuits) to enable both physical assistance and improved usability and acceptance for users. The XoSoft EU project has developed a modular soft lower limb exoskeleton to assist people with low mobility impairments. In this paper, we present the design of a soft modular lower limb exoskeleton to improve person's mobility, contributing to independence and enhancing quality of life. The novelty of this work is the integration of quasipassive elements in a soft exoskeleton. The exoskeleton provides mechanical assistance for subjects with low mobility impairments reducing energy requirements between $10 \%$ and $20 \%$. Investigation of different control strategies based on gait segmentation and actuation elements is presented. A first hip-knee unilateral prototype is described, developed, and its performance assessed on a post-stroke patient for straight walking. The study presents an analysis of the human-exoskeleton energy patterns by way of the task-based biological power generation. The resultant assistance, in terms of power, was $10.9 \% \pm 2.2 \%$ for hip actuation and $9.3 \% \pm 3.5 \%$ for knee actuation. The control strategy improved the gait and postural patterns by increasing joint angles and foot clearance at specific phases of the walking cycle.
\end{abstract}

*Corresponding author. E-mail: christian.dinatali@iit.it 
KEYWORDS: Soft exoskeleton; Exosuit; Robotic wearable device; Quasi-passive actuation; Legged locomotion; Gait assistance.

\section{Introduction}

The population of the European Union in 2016 was estimated at 510.3 million, of which $19.2 \%$ were aged 65 years and older. This represents an increase of $0.3 \%$ from the previous year, and $2.4 \%$ over the previous 10 years. By 2080, 29.1\% of the population will be aged $65+$. The growth in the relative share of older people may be explained by increased longevity, a pattern that has been apparent for several decades as life expectancy has risen. ${ }^{1}$ Gait impairments and the need for mobility assistance are commonly observed in people with different health conditions, such as general agerelated frailty, incomplete spinal cord injury, and residual paresis after stroke. ${ }^{2}$ It can be expected that the number of people in need of low-to-moderate mobility assistance will continue to increase. This brings considerable attention on how to provide assistance for the elderly in their daily life, especially concerning mobility. Thus, there is the opportunity to use exoskeletons to provide physical assistance to address mobility assistance. ${ }^{3}$

The literature indicates that some exoskeletons address the needs of elderly people by enhancing stamina and reducing effort to accomplish daily tasks. ${ }^{4}$ In fact, several devices have been developed to facilitate the achievement of different tasks such as walking, sitting, and stair-climbing by providing additional power for people with muscular weakness.

Some of the traditional wearable devices are developed as parallel robotic legs connecting the user's waist and their feet through kinematic chains. One of these is the XoR prototype, developed for postural control of elderly people and persons with mobility disability. ${ }^{5,6}$ This design is actuated in a hybrid configuration, combining pneumatic artificial muscles and electric motors together. The exoskeleton structure is composed of two full legs and a trunk module, which all together weigh $33 \mathrm{~kg}$. It also provides gravity compensation. However, it has only been demonstrated in simulations.

Moving towards the device strategy of encumbrance and weight reduction, exoskeletons, generally, do not have configurations with fully actuated lower limbs, but rather a further simplification is adopted. The exoskeleton configuration introduced in ref. [9] presents only knee actuation, where pneumatic muscles are employed while the ankle joint is kept passive. Similarly the $\mathrm{WWH}^{8,9}$ prototype is presented. In this case, the hips and knees are actuated by traditional gear transmissions, motors, and prismatic actuation. Both examples detailed above have been designed for the elderly to provide gravity compensation, and also to assist walking, climbing stairs, and sitting activities. The analysis of electromyography signals presented a muscular activity reduction of $30 \%$ and $40 \%$, respectively.

When exoskeletons are designed to address the needs of elderly and people with motion disability, a further device simplification is required. An example of navigation aid has been introduced in ref. [10] where a slim design and size reduction have been achieved. The unilateral actuation system is based on motor assistance for the knee and ankle, transmitted by employing flexible shafts and worm gears. The mechanical transmission is developed along the user-assisted leg, keeping the system unburdensome, while the actuation system is contained in a backpack. Walking experiments have been carried out with both healthy subjects and paralyzed subjects, performing an average assistance of $20 \%$. The tendon-driven exoskeleton system EXPOS ${ }^{11}$ is another example of weight reduction of the outline configuration and equipment to meet user needs. The system is accompanied by a smart caster walker, which carries part of the heavier components such as motors, drivers, controllers, and batteries. Thus, the wearable device is lightweight and simple, with a weight of $3 \mathrm{~kg}$. An elderly subject executed sitting exercises with a $32 \%$ assistance of muscular power.

Since the new generation of exoskeletons for the elderly must address not only the task of assistance but also acceptance and usability, some research groups proposed the evolution of exoskeletons to exosuits. The exosuit introduced in ref. [12] supports the ankle joint in plantar and dorsi-flexion in one leg and weighs about $4 \mathrm{~kg}$ in total. The system exhibited a $32 \%$ reduction of metabolic cost for post-stroke walking by delivering a biological joint torque assistance of $12 \%$. A similar approach for knee assistance is presented in ${ }^{13}$ using a bilateral configuration of $3 \mathrm{~kg}$ without a battery. During the straight walking swing phase of elderly subjects it showed a reduction of $7 \%$ of energy expenditure by applying $8 \%$ of hip torque. Finally, the Myosuit in ${ }^{14}$ combined active and passive elements assisting 

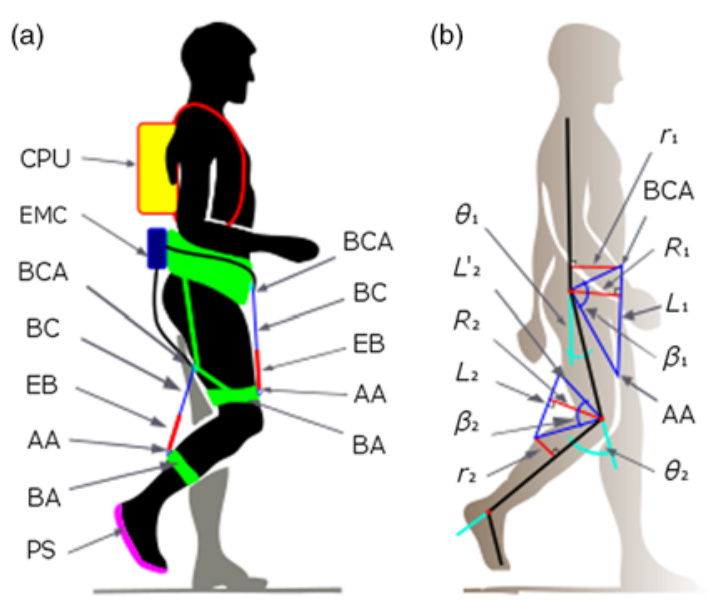

Fig. 1. (a) Conceptual drawing of the XoSoft Beta 1 prototype displaying the technologies integrated on: Actuator Attachment $(A A)$, Body Attachment $(B A)$, Bowden Cable $(B C)$, Bowden Cable Attachment $(B C A)$, Elastic Band $(E B)$, Electromagnetic Clutch $(E M C)$, Pressure Sensor $(P S)$. (b) Geometrical representation of the actuation units. The abbreviations are presented in Table I.

the user with a system weight of $4.6 \mathrm{~kg}$. The system shares a rigid structure embedded on a suit that interfaces with the user. The overall system takes advantage of a synergy-based approach for assistance. The assistance resulted in $26 \%$ of the knee moment and up to $35 \%$ power reduction on sitting tasks. These examples take the benefit of a soft wearable structure to reduce the burden of the device. On the other hand, by using active electrical motors combined with cable or belt to actively transmit power to the assisted joint, weight, power consumption, and encumbrance due to batteries and other mechanical components become an issue.

The recent trend in exosuit design is to avoid bulky, rigid, heavy exoskeletons by targeting light, soft, and wearable devices. Thus, system autonomy, usability, and acceptance became the foundation of the XoSoft project. The aim of the project is to develop a soft, modular, bio-mimetic, and quasipassive exoskeleton that is intended to assist users with low-to-moderate mobility impairments. ${ }^{15}$ It also employs user-centered design approach to meet user requirements. ${ }^{16}$ The design targets the assistance of the lower limbs mainly for elderly, post-stroke, or partial spinal cord injury subjects.

The XoSoft Beta 1 prototype presents a quasi-passive actuation ( $Q P A)$ strategy as a result of system optimization and simulation framework introduced in ref. [17]. The system does not provide any active force, and it is therefore completely passive. If needed, it has the ability to deactivate the assistive force. A preliminary XoSoft version, a single hip assistance module, has been tested, proved, and validated on a subject as presented in ref. [18].

The contribution of this work stems from the introduction of a soft wearable device Beta 1 prototype, for gait assistance of elderly people, by employing $Q P A \mathrm{~s}$ in a unilateral configuration. Particularly, the wearable device is based on assistance of hip and knee flexion. The system is illustrated theoretically and practically, and a validation section has been conducted to demonstrate its effectiveness. An extensive energy analysis of the human-exoskeleton interaction in relation to taskbased biological power generation is conducted. In Section 2, the methods and design implementation are presented. In Section 3, the experimental protocol and results are explained and discussed. In Section 4, conclusions and future developments are addressed.

\section{Design and Methods}

The prototype Beta 1 developed within the XoSoft project is composed of a unilateral hip and knee configuration. The actuation principle is defined as $Q P A$ and it is composed of an elastic band (EB) and a clutch configured in series. $Q P A$ refers to any controllable element that cannot apply a nonconservative motive force. ${ }^{19}$ Thus, $Q P A$ includes any combination of variable-dampers or clutches in conjunction with passive components such as springs. The work presented in ref. [18] introduces the modular $Q P A$ unit for a single hip in a soft wearable device, the modular design description and a preliminary validation. The actuation design main idea and configuration is based on the system optimization introduced in ref. [17]. As previously mentioned, the Beta 1 prototype was developed through a user-centered design approach. Therefore, it is meant to be easy to wear, soft, and comfortable. The prototype has been designed and tailored for a participant with lower limb mobility 
Table I. Abbreviations.

\begin{tabular}{|c|c|}
\hline Acronym & Meaning \\
\hline$Q P A$ & Quasi-passive actuation \\
\hline$P A$ & Passive actuation \\
\hline$A A$ & Actuation attachment \\
\hline$B A$ & Body attachment \\
\hline$B C$ & Bowden cable \\
\hline$B C A$ & Bowden cable attachment \\
\hline$E B$ & Elastic band \\
\hline$E M C$ & Electromagnetic clutch \\
\hline$P S$ & Pressure sensor \\
\hline DoF & Degrees of freedom \\
\hline$D--H$ & Denavit-Hartenberg \\
\hline$\Lambda$ & Ratio of powers \\
\hline$C P U$ & Central processing unit \\
\hline$F S M$ & Finite state machine \\
\hline INX & Inactive XoSoft \\
\hline$G R F$ & Ground reaction forces \\
\hline$R A S I$ & Right anterior superior iliac spine \\
\hline LASI & Left anterior superior iliac spine \\
\hline RPSI & Right posterior superior iliac spine \\
\hline LPSI & Left posterior superior iliac spine \\
\hline$R K L E$ & Right knee lateral epicondyle \\
\hline$L K L E$ & Left knee lateral epicondyle \\
\hline$R K M E$ & Right knee medial epicondyle \\
\hline$L K M E$ & Left knee medial epicondyle \\
\hline$R A L M$ & Right ankle lateral malleolus \\
\hline$L A L M$ & Left ankle lateral malleolus \\
\hline$R A M M$ & Right ankle medial malleolus \\
\hline$L A M M$ & Left ankle medial malleolus \\
\hline$L I M$ & Left intermalleolus point \\
\hline RIM & Right intermalleolus point \\
\hline$L I C$ & Left intercondylar point \\
\hline$R I C$ & Right intercondylar point \\
\hline$S A C R$ & Sacrum bone \\
\hline$L R F$ & Left rear-foot at the ankle joint \\
\hline$R R F$ & Right rear-foot at the ankle joint \\
\hline LSHA & Left shank at the ankle joint \\
\hline RSHA & Right shank at the ankle joint \\
\hline LSHK & Left shank at the knee joint \\
\hline$R S H K$ & Right shank at the knee joint \\
\hline LTHK & Left thigh at the knee joint \\
\hline RTHK & Right thigh at the knee joint \\
\hline LTHH & Left thigh at the hip joint \\
\hline RTHH & Right thigh at the hip joint \\
\hline$L P E H$ & Left pelvis at the hip joint \\
\hline RPEH & Right pelvis at the hip joint \\
\hline
\end{tabular}

problem on his right side. In fact, the participant had experienced a stroke 7 years ago and presented a unilateral impairment of the lower and upper right extremities. At this stage, the control system uses a biomimetic approach based on gait segmentation to properly activate or deactivate the $Q P A$ in order to alternate storing and releasing actuation phases. The control exploits insole sensors feedback to identify gait patterns, consequently determining assistance. Figure 1(a) shows the Beta 1 conceptual drawing where technical components and their disposition are presented.

\subsection{Mechanical model}

The system, composed of lower limbs, can be considered as a set of rigid links. We assume that there is no linear translation in the joints but only rotational motion. Thus, it is possible to analyze human motion according to the assumption of continuum mechanics. The approximation adopted in this 
Table II. $D--H$ parameters.

\begin{tabular}{lcccccc}
\hline Joint & $\boldsymbol{\gamma}_{\boldsymbol{i}}$ & Number & $\boldsymbol{\alpha}_{\boldsymbol{i}}$ & $\boldsymbol{a}_{\boldsymbol{i}}$ & $\boldsymbol{d}_{\boldsymbol{i}}$ & $\boldsymbol{\theta}_{\boldsymbol{i}}$ \\
\hline Base & 0 & $1_{0 \rightarrow 1}$ & $\pi / 2$ & $a_{0}$ & $p_{0}$ & 0 \\
Hip & $-30^{\circ} / 120^{\circ}$ extension-flexion & $2_{1 \rightarrow 2}$ & $\pi$ & $l_{1}$ & $d_{0}$ & $\gamma_{1}-\pi / 2$ \\
Knee & $0^{\circ} / 150^{\circ}$ extension-flexion & $3_{2 \rightarrow 3}$ & $\pi$ & $l_{2}$ & 0 & $\gamma_{2}$ \\
Ankle & $-40^{\circ} / 20^{\circ}$ plantarflexion-dorsiflexion & $4_{3 \rightarrow 4}$ & $-\pi / 2$ & $l_{3}$ & 0 & $\gamma_{3}+\pi / 2$ \\
\hline
\end{tabular}

Table III. Anthropometric measurements.

\begin{tabular}{lllc}
\hline Variable & $\begin{array}{l}\text { Measurement } \\
\text { (right leg) }\end{array}$ & $\begin{array}{l}\text { Measurement } \\
\text { (left leg) }\end{array}$ & Parameter \\
\hline Height & $1.7 \mathrm{~m}$ & & \\
Mass & $70 \mathrm{~kg}$ & & \\
Waist $\phi$ & $88 \mathrm{~cm}$ & & $p_{0}$ \\
Pelvis height & $90 \mathrm{~cm}$ & $15 \mathrm{~cm} / 15 \mathrm{~cm}$ & $a_{0} / d_{0}$ \\
Pelvis-Hip & $15 \mathrm{~cm} / 15 \mathrm{~cm}$ & $42 \mathrm{~cm}$ & $l_{1}$ \\
Thigh length & $41 \mathrm{~cm}$ & $38 \mathrm{~cm}$ & $l_{2}$ \\
Shin length & $38 \mathrm{~cm}$ & $22 \mathrm{~cm}$ & $l_{3}$ \\
Foot length & $22 \mathrm{~cm}$ & & \\
\hline
\end{tabular}

(a)

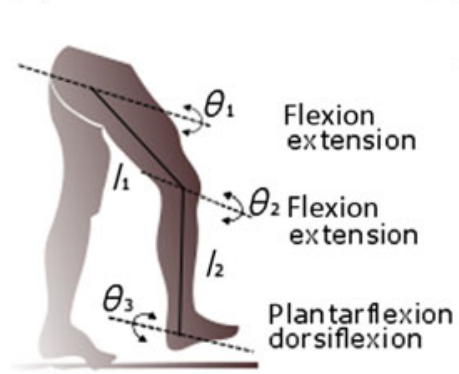

(b)

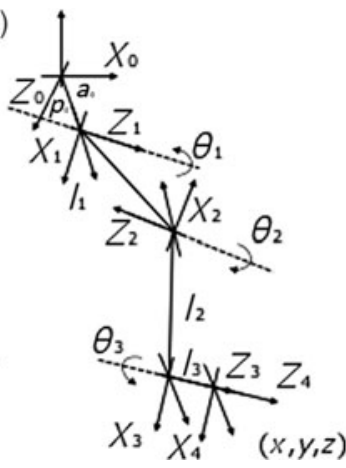

Fig. 2. (a) $D--H$ notation for lower limb. (b) Kinematic chain model.

work is that motion occurs only parallel to the sagittal plane. Therefore, each lower limb is analyzed as a three-joint configuration kinematic chain (hip, knee, and ankle) considering three degrees of freedom $(D o F)$. The lower limb model is presented in Fig. 2, and the pelvis is considered the center of the base frame $\left(X_{0}, Y_{0}, Z_{0}\right)$. The Denavit-Hartenberg $(D--H)$ parameters are presented in Table II, where the angles $\theta_{i}$ about axes $Z_{i}$ are variables depending on the range of motion $\gamma_{i}$ of each joint. The other two parameters, $a_{i}$ and $d_{i}$, are the body segment lengths, which are constants and dependent on the subject measurements (see Table III). Therefore, knowing the $D--H$ transformation matrix $T_{0}^{3}$ and the system generalized coordinates in the joint space $q=\left[\theta_{1}, \theta_{2}, \theta_{3}, 1\right]$, it is possible to obtain the end-effector (which in our case is the foot) time variant position, by applying direct kinematics. Equation (1) returns the time variant foot position as

$$
X(t)=T_{0}^{3} q(t)
$$

This consideration is used to analyze the exoskeleton effect on the subject's foot clearance.

The wearable device is developed to aid the user by applying a percentage of the needed power to accomplish the task. Thus, the exoskeleton is applying forces on user leg segments. The actuations embedded in the device are defined as $Q P A$ s and they are providing pulling forces between the attachments points $(A A$ and $B C A)$. As the attachments are below and a bove the user articulation, as shown in Fig. 1, the force generated by the $E B$ results coupled with the aided joint. Since the $E B$ is a passive element, this needs to be first elongated, and only then it can provide force. These two phases are referred to as storing and releasing phases. Therefore, the interaction with the user is important since the joint motion will actuate the $E B$ by providing elongation. The drawing in Fig. 1(b) summarizes the main mathematical elements related to the force/torque transmission. The initial cord 
length (series of $B C$ and $E B) L_{k}\left(\theta_{k}\right)$ is a function of the joint angle $\theta_{k}$, where the index ${ }_{k}$ assumes value $_{1}$ or 2 if referred to the hip joint or to the knee joint, respectively. The user's joint rotation generates an amount of elongation defined as $\Delta L_{k}$ on the cord. The total cord length is ruled by Eq. (2) as follows:

$$
L_{k}\left(\theta_{k}\right)= \begin{cases}L_{k}^{\prime} \sqrt{2-2 \cos \left(\beta_{k}\right)} & \text { if } \theta_{k} \text { is } \geq 0 \\ L_{0 k}+r_{k} \theta_{k} & \text { if } \theta_{k} \text { is }<0\end{cases}
$$

where $L_{k}^{\prime}$ is equal to $L_{k}^{\prime}=\sqrt{L_{0 k}^{2} / 4+r_{k}^{2}}$, and the angle $\beta_{k}$ is equal to $\beta_{k}=\pi-\theta_{k}-2 \arctan \left(\frac{2 r_{k}}{L_{k}}\right) . L_{0 k}$ is the length of the cord $B C$ and $E B$ in its neutral position such as when $\theta_{k}=0$. The torque provided by the actuation system is strongly influenced by the moment $\operatorname{arm} R_{k}\left(\theta_{k}\right)$ subtended by the cord $L_{k}$, and by the cord elongation $\Delta L_{k}$. The cord elongation is completely supported by the $E B$, whereas the $R_{k}\left(\theta_{k}\right)$, function of the joint angle, is expressed as in Eq. (3).

$$
R_{k}\left(\theta_{k}\right)= \begin{cases}r_{k} & \text { if } \theta_{k} \text { is }<0 \\ \frac{L_{k}^{\prime 2}}{L_{k}^{\prime} \sqrt{2-2 \cos \left(\beta_{k}\right)}} \sin \left(\beta_{k}\right) & \text { if } \theta_{k} \text { is } \geq 0\end{cases}
$$

As a first approximation, $R_{k}\left(\theta_{k}\right)$ is considered to be always aligned to the sagittal plane. Therefore the torque generated on the assisted joint is proportional to the $E B$ 's force $f_{k}$ as Eq. (4).

$$
\tau_{k}=R_{k}\left(\theta_{k}\right) f_{k}=R_{k}\left(\theta_{k}\right) K_{E B} \Delta L_{k}
$$

Where $K_{E B}$ is the $E B$ 's constant.

The task conducted by the subject wearing the soft exoskeleton is characterized by a particular amount of torque $\tau_{t}$ for each joint. The exoskeleton aids the user by providing the torque $\tau_{e x}=\left[\tau_{1}, \tau_{2}\right]$, as it is defined in Eq. (4). The mechanical system, comprising of both user and exoskeleton, requires an amount of energy for the storing phase. This energy is provided by the user during determined gait phases. Then the total amount of absorbed energy is returned by the exoskeleton in the form of assistance. During the releasing phase, the $E B s$ are providing torque on the aided joints.

The reason behind this work is to design and control the actuation system of the soft wearable device. As the typology of the actuation is based on the energy exchange between $Q P A$ and the leg segment, it is fundamental to minimize the energy requirements during gait. ${ }^{20}$ In order to analyze the system behavior, the power associated with the task, as the combination of the torque and angular velocity of each joint, is taken into consideration as in Eq. (5).

$$
P_{t}=\tau_{t} \dot{\theta}_{t}
$$

Muscles generate and absorb the mechanical energy necessary to accomplish the movement that we observe. A good estimation of the muscle activity while wearing the exoskeleton could be predicted by the mechanical power. Indeed, a negative value of mechanical power is related to the muscle activity of absorption provided by concentric contraction, whereas a positive value with generation of energy by eccentric contraction.

Moreover, it is possible to calculate the assistance provided by the prototype Beta 1 computing the ratio of powers $(\Lambda)$ as

$$
\Lambda=\left|\frac{P_{t}-P_{e x}}{P_{t}}\right|
$$

where $P_{t}$ is the measured power of the user not wearing the exoskeleton. $\Lambda=1$ means that the prototype is not providing any assistance, while $\Lambda=0$ represents $100 \%$ of assistance.

\subsection{Design description}

2.2.1. Garment. The garment of Beta 1 prototype has been designed mainly focusing on the user needs (such as "donning"I and "doffing"). When developing a fabric suitable for the prototype, the following properties were taken into account: fiber composition, weight, elongation/stretch, absorbency/wetting, and comfort. Wear comfort is a complex phenomenon, ${ }^{21}$ but in general, it can be divided into four different main aspects: 
- Thermos physiological wear comfort, as it directly influences a person's thermoregulation. It comprises heat and moisture transport processes through the clothing. Key factors to consider include thermal insulation, breathability, and moisture management.

- The skin sensorial wear comfort characterizes the mechanical sensations, what a textile causes at direct contact with the skin. These perceptions may be pleasant, such as smoothness or softness, but they may also be unpleasant, if a textile is scratchy, too stiff, or clings to sweat-wetted skin.

- The ergonomic wear comfort deals with the fit of the clothing and the freedom of movement it allows. The ergonomic wear comfort is mainly dependent on the garment's pattern and the elasticity of the materials.

- The psychological wear comfort is relevant too. It is affected by fashion, personal preferences, ideology, etc.

In order to cope with these aspects, the trousers are tailored for the subject. The selected fabric is lycra for the stretchable parts, whereas polyester webbings have been used to guarantee enough inelastic response on the $B A$ due to force applications. Lateral zips have been sewed to facilitate quick donning and doffing procedures. A safety harness belt (CE 01, Tractel, France), equipped with a safety handle to prevent falls, was mainly used to anchor $B C \mathrm{~s}$ and $E M C \mathrm{~s}$. The overall weight of the garment is $0.680 \mathrm{~kg}$ and $2.2 \mathrm{~kg}$ for the safety harness belt equipped with the actuation units $(0.7 \mathrm{~kg}$ each).

2.2.2. Actuation units. The actuation units are designed to be quasi passive, modular, and lightweight. Thus, it is possible to easily change leg segment and the physical actuation features by changing the $E B$. The Beta 1 prototype has two QPAs, the first one is dedicated to the hip flexion while the second one is for the knee flexion, as shown in Fig. 1(a) and (b). The off-the-shelf parts composing the system are an electromagnetic clutch (111-06-13G, Miki Pulley Co. Ltd, Kanagawa, Japan) and $E B$ s of different stiffness (Loop Band, Fit Point, Brescia, Italy). A $B C$ (link cable, Misumi Group Inc., Tokyo, Japan) connects each $E M C$ with the corresponding $E B$, and it is used to mechanically transmit the $E M C$ status (engaged or disengaged) to the $E B$. Therefore, we consider the series of $E B$ and $B C$ being the selected physical element used to store and release mechanical power based on its geometrical features as displayed in Fig. 1(b). If the clutch is not engaged, this mechanical element could also be neutral to the system. Thus, no storing or releasing phase is involved while the user is moving. As a geometrical consideration, the attachment positioning of $A A$ and $B C A$ with respect to the leg segment is very important. The two attachments must be above and below the actuated joint.

Figure 3 represents the schematic of the actuation unit including $E M C$ and $B C$. This system is mainly characterized by two different configurations defined by the $E M C$ status. When the clutch is engaged, it blocks the length of the system composed by the series of $E B$ and $B C$. Hence, the $B C$ is not free to travel anymore. The initial cord length $L_{k}\left(\alpha_{k}\right)$ is subjected to the cord elongation $\Delta L_{k}$ as the user's joint is rotating. The torque provided by the actuation system is strongly influenced by the moment arm $R_{k}\left(\alpha_{k}\right)$ subtended by the cord $L_{k}$, and by the cord elongation $\Delta L_{k}$. Thus, the system is storing and delivering energy as a consequence of the joint rotation. During the first phase of storing, a resistance torque is determined and the user has to win it. Then, as soon as the joint starts rotating in the opposite direction, the system releases the accumulated mechanical energy. During this phase, the system is providing assistance to user motion until the leg segment returns to its initial engage position. Therefore, according to the gait phase and the clutch engagement timing, it is possible to store and deliver different amounts of energy.

When the clutch is disengaged, unappreciated force is exerted by the system to the user and it results being transparent. Also, the $B C$ extremity connected to the clutch is fixed to a recall elastic, which is characterized by the stiffness constant $K_{c}$ as expressed in Fig. 3. Thus, it keeps the series of $B C$ and $E B$ always tight during the movement. In this state, the clutch is not blocking the system, the $E B$ is not stretching, and the $B C$ is free to travel according to the hip rotation.

2.2.3. Shoe insole. A pair of custom insoles embedded with force sensors are used to detect contact with the ground. Four sensors are located inside the insole, one under the heel, two under the metatarsal area, and one under the phalanges. The real-time information is used to segment the gait path to identify the different events of the user gait: heel strike, flat foot, and toe off. Consequently, 
(a)

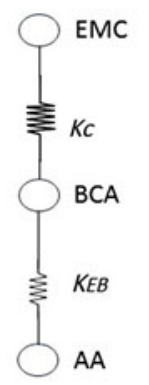

Clutch engaged $K C \gg K E B$

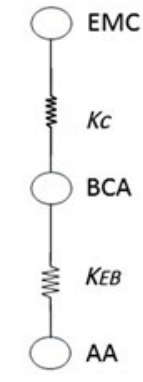

Clutch dis-engaged $K c \ll K E B$ (b)

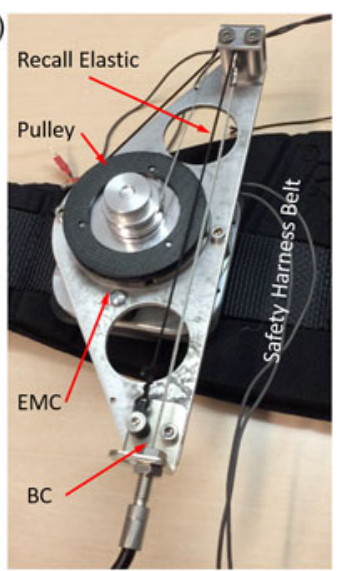

Fig. 3. (a) Mechanical schematic of the actuation unit. (b) Physical representation of the $E M C$ and recall elastic.

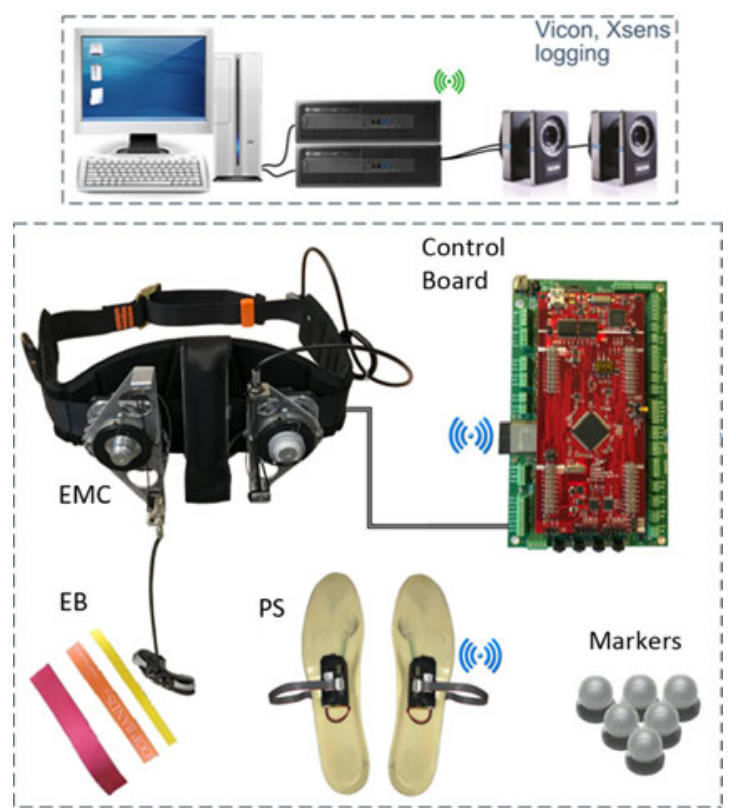

Fig. 4. System overview of the test assessment. On the top: the instrumentation used for motion capture. On the bottom: markers and main hardware components of the exoskeleton described in Section 2.2.

specific control signals are applied based on three phases of the gait. As for the sensorized insole described in ref. [22], a custom insole was cast with soft foam (Hekapur PU, Exact Plastics, Bröckel, Germany), which included four force sensing resistors (FSR, 1-Inch ShuntMode, Sensitronics, Bow, USA) (Fig. 4).

The PSs are used as switches providing a digital signal dependent on whether a pressure threshold is overcome. Figure 5 shows the digital signal of the heel, mid foot, and toe PSs output as a function of the gait.

2.2.4. Electronics and communication. The electronics consists of two Wi-Fi modules (ESP32, Espressif, Shangai, China) transmitting to the main CPU board (Launchpad XL Development Kit, Texas Instruments, Dallas, US) with a custom shield board providing power management, I/Os (Input/Output), and CAN (Connected Area Network) Communication as displayed in Fig. 4. Each ESP32 wirelessly communicates the FSR (Force Sensing Resistor) signals to the $C P U$. The $C P U$ then segments the gait phase, which is represented as a logic state of a FSM (shown in Fig. 5) on the main control algorithm. In each state, then, power outputs to the clutches are set or reset.

The electronics and batteries are embedded in a backpack with total weight of $2 \mathrm{~kg}$. 


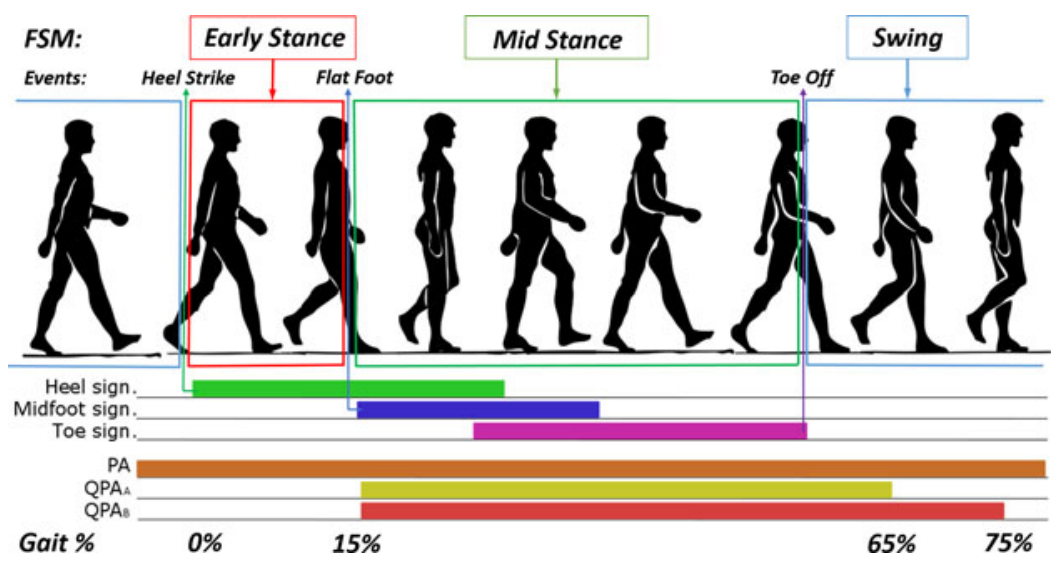

Fig. 5. Schematic of the insole sensor output, FSM, and control strategies for the right leg.

2.2.5. Control strategy. Since the actuation strategy is a $Q P A$ or a passive actuation $(P A)$, the overall effect of the XoSoft prototype is strongly affected by the control actuation and the interaction with human action.

The storing phases, corresponding to the $E B$ elongation, should be in conjunction with the stance phase. In this way, the user muscular activity must be greater than the resistance offered by the $E B$ elongation. At the same time, however, it takes advantage of body dynamic inertia that is helping the user winning the elastic resistance.

Based on the signal output of the shoe insole, the control segmentation can determine the three gait phases (early stance, mid stance, and swing), as presented in Fig. 5. The control strategy activates the clutch storing energy. Before the disengage of the clutch occurs, user motion will lead the transition from storing phase to releasing phase. Therefore, by controlling the timing of the clutch activation, it is possible to modulate the assistance. In other words, the engagement timing together with the user motion contributes to the generation of the aid torque by modulating the $E B$ elongation lengths.

In this work, three different control strategies are implemented.

- Clutches always engaged, resulting in a $P A$.

- Clutches activated at mid stance ( $\sim 15 \%)$ of the gait and deactivated swing after toe off ( $\sim 65 \%$ of gait), $Q P A_{A}$.

- Clutches activated at mid-stance $(\sim 15 \%)$ of the gait and deactivated during swing after toe off ( $\sim 75 \%$ of gait), $Q P A_{B}$.

The proposed control strategy $Q P A_{A}$ and $Q P A_{B}$ are achieved by applying two different delays after toe off, of 0.1 and $0.2 \mathrm{~s}$, respectively. The logic behind the hip actuation strategy is presented in a previous work, ${ }^{18}$ which is the result of the optimization process. ${ }^{17}$

Figure 6 represents knee torque as a function of the knee angle during gait, where the swing phase is characterized by a large angular displacement, and the stance phase is represented by the curve between heel strike and beginning of swing. The chart also shows the instant of clutch engagement for the knee actuation for each of the three control strategies (15\% of gait). The clutch engagement is represented by the region of angle inferior to the mid stance event (yellow vertical line). Therefore, the knee range of angle related to the storing and releasing phases is also represented. The control strategy $Q P A_{A}$ is performing only the storing and releasing phase I, whereas the $P A$ is also operating during storing and releasing phase II. The $Q P A_{B}$ is performing during storing and releasing phase I. Since the clutch is engaged until $75 \%$ of gait (about the end of swing phase), the knee angle results are inferior to the mid stance angle; thus, the $Q P A_{B}$ performs some of the storing phase II.

Conceptually the storing-releasing phase I contributes to enlarge the swing range of motion, and thus the release of energy is coherent with the joint motion. On the other hand, the storing-releasing phase II is mainly used to decrease the knee hyper-extension by using the actuation system as a shock absorber. 
Table IV. Third-order polynomial fitting coefficients.

\begin{tabular}{lccc}
\hline Band & $\boldsymbol{a}_{\mathbf{3}}$ & $\boldsymbol{a}_{\mathbf{2}}$ & $\boldsymbol{a}_{\mathbf{1}}$ \\
\hline $\mathrm{A}$ & $9.06 * 10^{-6}$ & -0.0049 & 1.1941 \\
$\mathrm{~B}$ & $1.22 * 10^{-5}$ & -0.0066 & 1.6208 \\
\hline
\end{tabular}

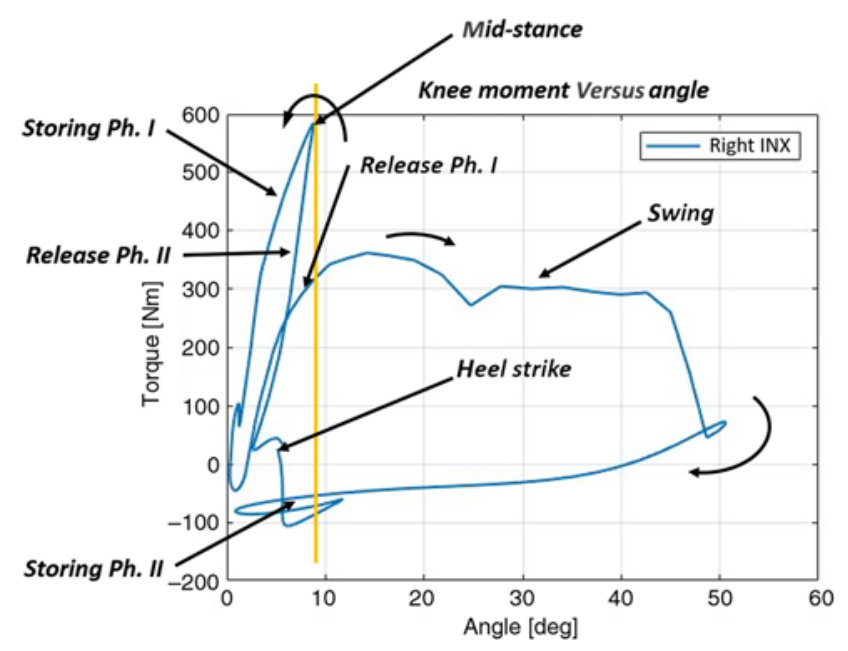

Fig. 6. Moment versus angle for the right knee gait.

\section{Experimental Section}

\subsection{Experimental protocol}

The system hardware has been characterized before the assessment on a subject. ${ }^{18}$ The mechanical and electrical features of the actuation unit have been evaluated by testing both $E B$ and $E M C$. Two different $E B s$ are integrated in the $Q P A$. The first $E B_{A}$ has a nominal elastic coefficient of $700 \mathrm{~N} / \mathrm{m}$ and the second $E B_{B}$ of $950 \mathrm{~N} / \mathrm{m}$. Since the stiffness characteristic of these latex $E B s$ is not linear, and in order to estimate the elastic force $F(x)$, a third-order polynomial has been used to fit elastic behavior as a function of the elongation percentage $(x)$, as in equation:

$$
F(x)=a_{3} x^{3}+a_{2} x^{2}+a_{1} x
$$

Table IV reports the interpolation parameters.

The right knee and hip modules of the wearable exoskeleton are demonstrated on a 68-year-old male (mass: $72 \mathrm{~kg}$; height: $170 \mathrm{~cm}$ ) who experienced a cerebrovascular stroke in 2011 . The subject presented unilateral gait impairment on his right side. The subject (shown in Fig. 7) performed ten straight line walking trials for each of the seven tested condition (investigation protocol BASEC-Nr. 2016-01406). First, the condition with inactive INX was tested. In this condition, all components of Beta 1 prototype were worn but the control was deactivated. Thus, the device was inactive and almost transparent to the user. Then the three control strategies presented in Section 2.2.5 were evaluated. All the control strategies have been tested by using two $E B s$ : the $E B_{A}$ and $E B_{B}$. In order to avoid any unexpected effects related to subject fatigue, two minutes of pause were observed between the trials.

Retroreflective markers were applied to different body segments (forefoot, rear-foot, shank, thigh, pelvis, and trunk) and recorded by a 3D high-speed camera system with 12 cameras (Vicon Vantage V5, Vicon Motion Systems Ltd, Oxford, UK) with a sampling rate of $240 \mathrm{~Hz}$. Two markers were also applied to the ends of the EBs to monitor their length during the test. The motion capture was collected in combination with two AMTI force plate sensors (OR6-7-2000, AMTI Inc, Watertown, US) to record GRF at $1200 \mathrm{~Hz}$. The speed was kept constant during all the trials; consequently, the step size was consistent aside from some natural variability from step to step. There are various factors that may influence the accuracy of marker-based motion capture. One of the notable negative effects is due to soft tissue underneath marker resulting in marker movement not representing underlying movement of bone. To mitigate the negative effects, four tracking markers were used for each 


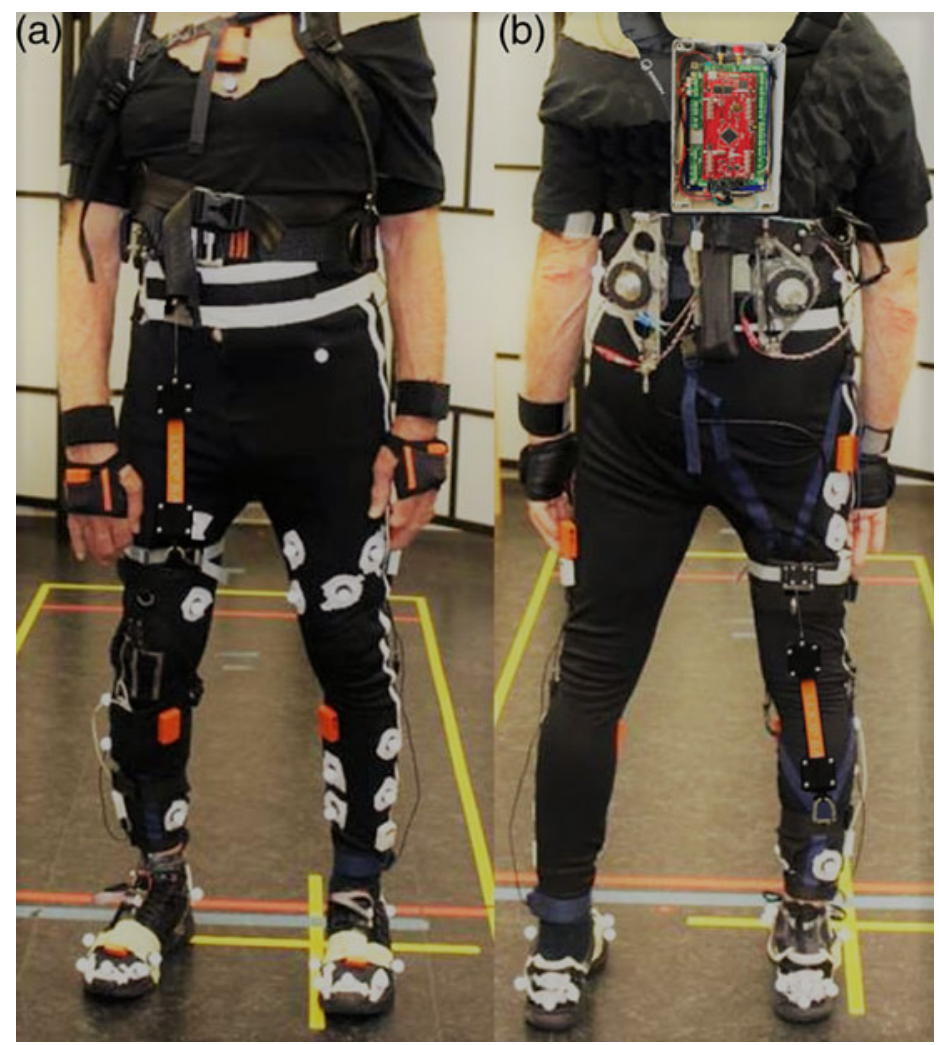

Fig. 7. Participant wearing XoSoft: (a) front and (b) back view.

segment, and an optimization approach (singular value decomposition ${ }^{23}$ ) was used to minimize the effect of relative marker movements within one segment.

Joint angles were calculated based on the standards defined by the International Society of Biomechanics. ${ }^{24}$ The description for kinematics and dynamics calculations applied on stereometric data is provided in the appendix Sections A.1 and A.2. All kinematic data are plotted from $0 \%$ to $100 \%$ of gait cycle ( $0 \%$ is the heel touch-down, whereas $100 \%$ corresponds to the consecutive touch-down of the same heel). Then, dynamic analysis was performed to extract forces and torques at the user's lower limbs articulation level.

One of the objective of this paper is to evaluate the design of Beta 1 prototype that allows energy exchange between the wearable device and the user's hip and knee joints, thus reducing the energy requirements during gait. Equations (4) and (5) are used to display the energy storing and releasing phases during gait under the condition of $P A, Q P A_{A}$, and $Q P A_{B}$ and then compared with INX. Equation (6) is applied to evaluate the assistance provided by Beta 1 prototype under the above conditions.

Postural improvements of the user wearing the device are also analyzed. In particular, foot clearance, measured as difference between the ankle joint spatial position during mid-swing (instant when the knee reaches maximum flexion) with respect to the INX condition, is evaluated.

\subsection{Results: postural considerations}

Figure 8(a) and (b) show, in red, the user's right hip and knee angles, respectively, INX. The overall effect of Beta 1 prototype under the control conditions of $P A, Q P A_{A}$, and $Q P A_{B}$ combining also the $E B_{A}$ and $E B_{B}$ are displayed in green for the left side and in blue for the right side of hip and knee angles. These green and blue trends are presented as average and standard deviation (bandwidth) of the six conditions analyzed. The INX left angles of hip and knee are omitted since the trends are within the green bandwidth.

The charts (Fig. 8(a) and (b)) clearly present the user's postural compensation on his left side due to the right-side impairment. Thus, the user's left side has higher range of motion and gait parameter asymmetry on both hip and knee angles if compared with the right side. 

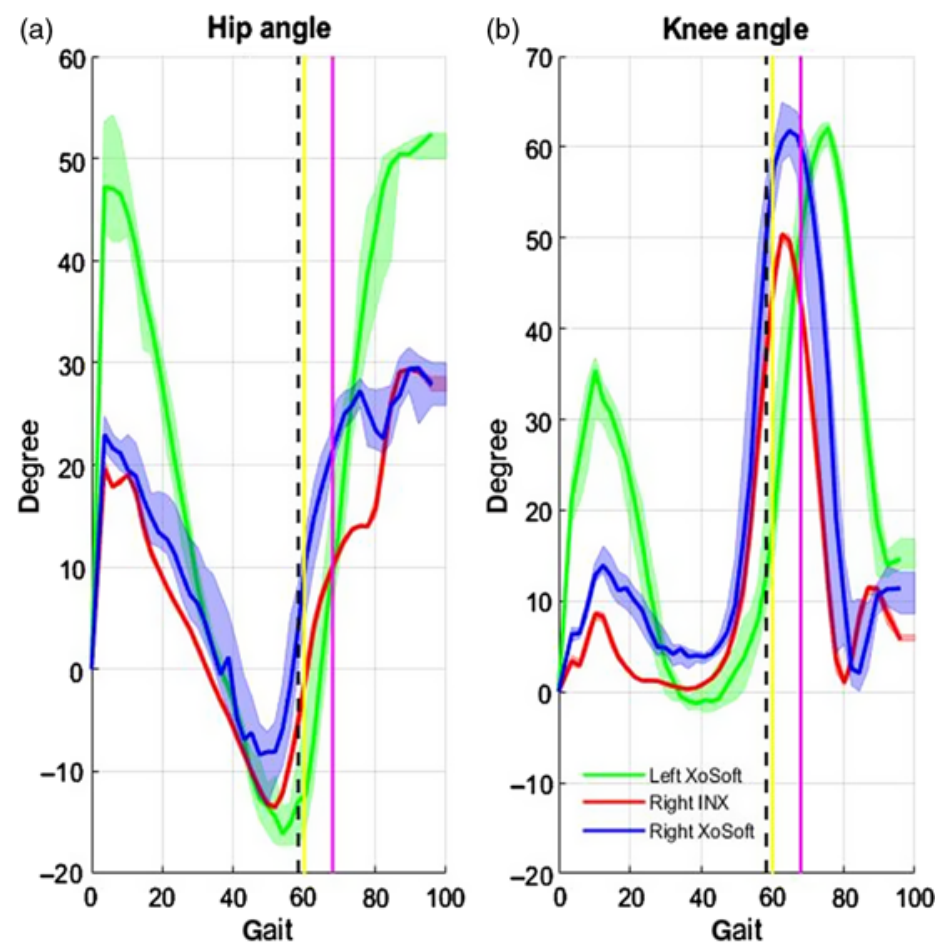

Fig. 8. Comparison of hip (a) and knee (b) joint angles of the user's left leg (healthy side). The right leg angles are displayed under the condition of wearing XoSoft and INX. The vertical lines represent the instant when toe off occurs for the three trends: left XoSoft (pink), right XoSoft (yellow), and right INX (black).

The overall benefit of Beta 1 prototype shows an augmentation of hip and knee angle values about $70 \%$ of the gait. This benefit occurs about $10 \%$ of the gait after the right toe off. Also range of motion is slightly modified towards the left reference and the gait asymmetry is apparently reduced.

Figure 9 shows force and moment applied by the $E B_{A}$ and $E B_{B}$ under the $P A, Q P A_{A}$, and $Q P A_{B}$ conditions for the knee and hip. Again, the trends of force and torque are presented as average and standard deviation (bandwidth) of the six conditions analyzed. The geometrical model used to calculate the effect of XoSoft on the user was presented in Section 2 by employing Eqs. (2)-(4) and (7). Figure 9(a) represents the actuation generated on the user's right hip. The bandwidth mainly corresponds to the delay of initial actuation, such that $P A$ is activated since $0 \%$ of the gait (heel strike) $Q P A_{A}$ and $Q P A_{B}$ from about $15 \%$ of the gait (midstance). Then, the system is engaged until $65 \%$ or $75 \%$ as presented in Fig. 5. Strategy $P A$ has the clutches activated until $70 \%$ of gait; after that the $E B$ becomes slack because the joint angle results being higher than the initial angle. In fact, when the hip joint angle reaches the same angle value it has at $0 \%$ of gait, the $E B$ elongation is null. Figure 9(b) shows the force and torque generated on the knee under all the same conditions analyzed. Since the clutch activation (shown in Fig. 5) is provided at $15 \%$ of the gait for all control strategies, assistive force is generated if the knee angle (Fig. 8(b)) remains inferior to the value assumed at the activation instant. If the angular value is greater than the activation angle (at $15 \%$ of gait), the system is slack not transmitting any forces on the knee joint. Therefore for the $Q P A_{A}$ and $Q P A_{B}$, the average activation range is between $15 \%$ and $50-60 \%$ of the gait. The $P A$ presents the same range of activation plus the final part of the swing (70-90\%). Also $Q P A_{B}$ presents a small section of activation within the range of gait of $70-75 \%$. The activation within the gait range from $15 \%$ to $60 \%$ is releasing an amount of torque which is augmenting the maximum flexion at mid-swing as displayed in Fig. 8. The torque generated by the $E B$ at the end of the swing (70-90\%) acts as a damper to reduce the user's right knee hyper-extension due to the right-side impairment. From the overall behavior of all the six different control strategies, $Q P A_{B}$ strategy seems being not effective.

\subsection{Results: power considerations}

Figure 10(a) and (b) presents the comparison of gait-related power by using XoSoft or INX for hip and knee under the studied control strategies. As previously mentioned, the trends are presented as 
(a) Force and torque generated on hip

(b) Force and torque generated on knee
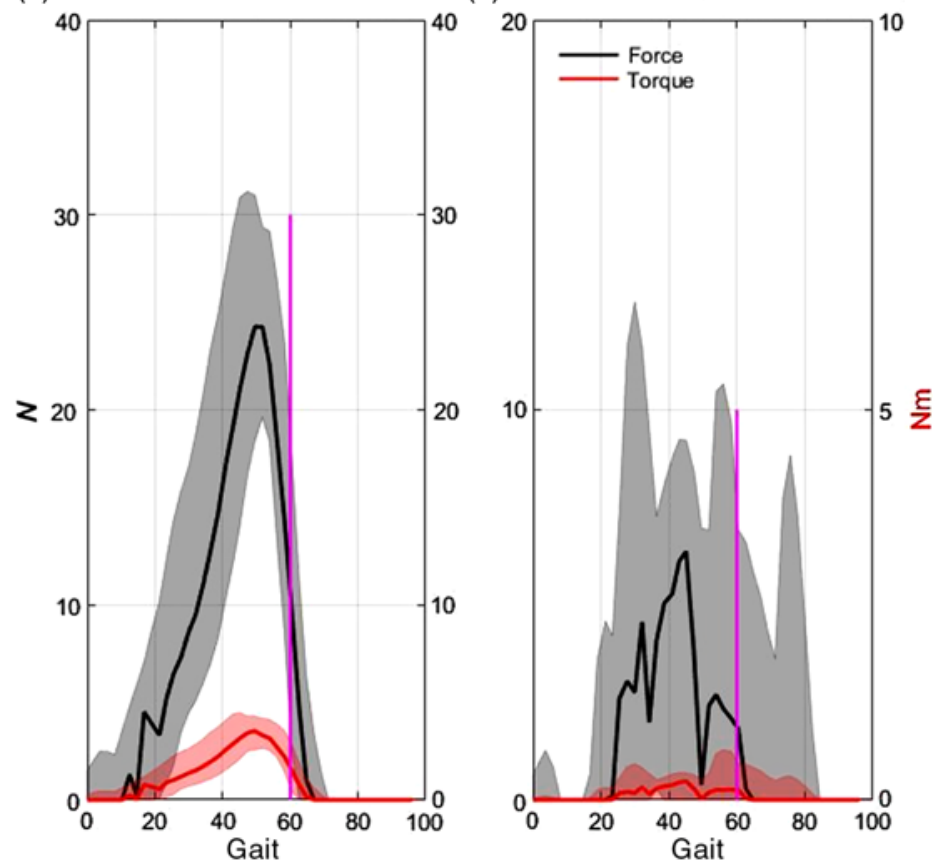

Fig. 9. Comparison of hip (a) and knee (b) joint force and torque generated on the right leg under the conditions $P A, Q P A_{A}$, and $Q P A_{B}$. The vertical pink line represents the instant when toe off occurs for the right leg.
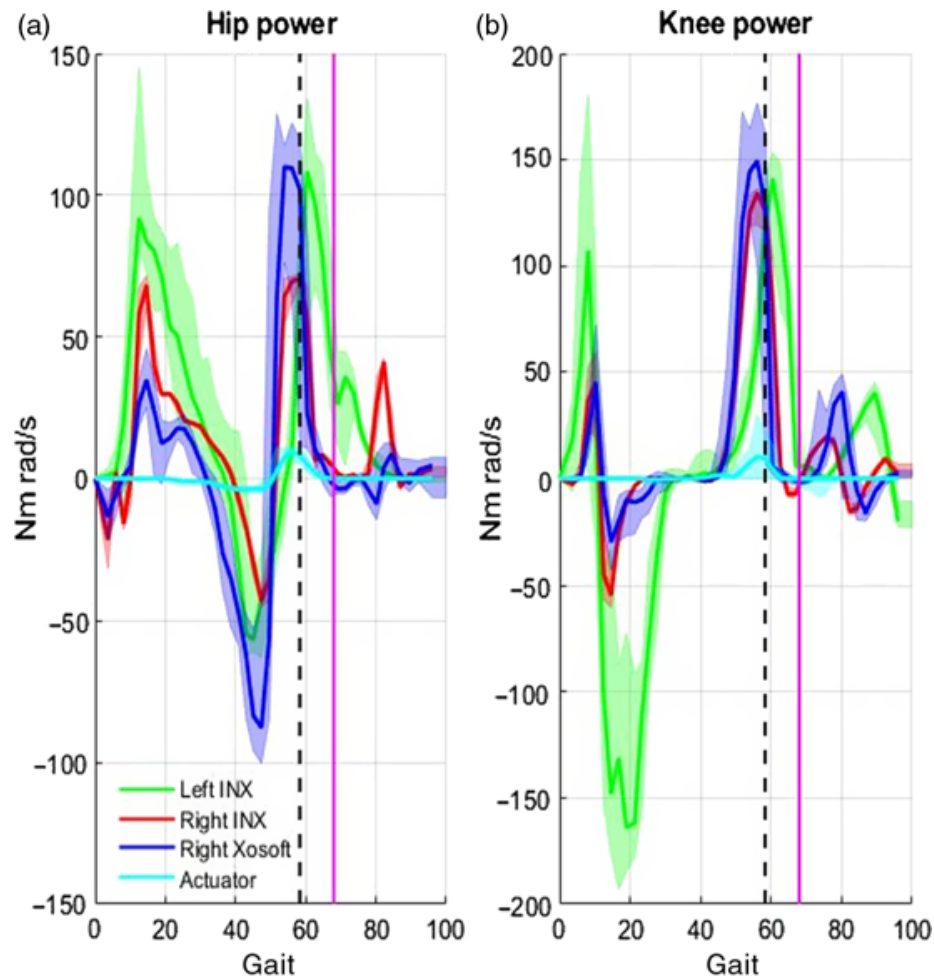

Fig. 10. Comparison of hip (a) and knee (b) joint power of left leg $I N X$, right leg with XoSoft, INX, and the power generated by the actuator under all the control conditions $\left(P A, Q P A_{A}\right.$, and $\left.Q P A_{B}\right)$ and different $E B s$. The vertical lines represent the instant when toe off occurs for left and right gait: left (pink), right (black). 
Table V. Ratio of powers $(\Lambda)$ and the storing/releasing XoSoft contribution power shared with the articulation under the following system conditions $P A, Q P A_{A}, Q P A_{B}$ and $E B_{A}, E B_{B}$.

\begin{tabular}{|c|c|c|c|c|c|c|}
\hline $\begin{array}{c}Q P A_{B} \text { and } \\
E B_{B}\end{array}$ & $\begin{array}{c}Q P A_{A} \text { and } \\
E B_{B}\end{array}$ & $\begin{array}{c}P A \text { and } \\
E B_{B}\end{array}$ & $\begin{array}{c}Q P A_{B} \text { and } \\
E B_{A}\end{array}$ & $\begin{array}{c}Q P A_{A} \text { and } \\
E B_{A}\end{array}$ & $\begin{array}{c}P A \text { and } \\
E B_{A}\end{array}$ & Condition \\
\hline 11.5 & 7.7 & 11.4 & 10.3 & 10.2 & 14.5 & $\Lambda$ hip \% \\
\hline 14.55 & 5.89 & 16.53 & 9.63 & 8.68 & 12.32 & $\%$ storing hip $(--)$ \\
\hline-8.28 & -15.87 & -10.72 & -6.45 & -12.15 & -14.61 & $\%$ storing hip $(-+)$ \\
\hline-14.47 & $\mathrm{NaN}$ & -2.02 & $\mathrm{NaN}$ & $\mathrm{NaN}$ & -6.37 & $\%$ releasing hip $(+-)$ \\
\hline 15.88 & 10.83 & 8.23 & 12.73 & 11.22 & 14.99 & $\%$ releasing hip $(++)$ \\
\hline 13.6 & 9.4 & 12.8 & 4.1 & 7.3 & 8.5 & $\Lambda$ knee $\%$ \\
\hline 20.95 & 10.91 & 17.60 & 3.13 & 4.28 & $\mathrm{NaN}$ & $\%$ storing knee $(--)$ \\
\hline-19.74 & -19.83 & -8.93 & -3.40 & -7.96 & -8.12 & $\%$ storing knee $(-+)$ \\
\hline-32.30 & -12.23 & -13.39 & -0.77 & -4.15 & -10.13 & $\%$ releasing knee $(+-)$ \\
\hline 6.38 & 4.49 & 14.06 & 7.06 & 7.99 & 6.12 & $\%$ releasing knee $(++)$ \\
\hline
\end{tabular}

average and standard deviation (bandwidth) of the six conditions analyzed ( $P A, Q P A_{A}$, and $Q P A_{B}$ combined with $E B_{A}$ and $E B_{B}$ ). The power is calculated exploiting Eq. (5). In particular, the blue trend represents the power $I N X$ of both joints for the affected leg. The difference with the left side, represented in green, is clear and is due to the subject's postural compensations. The light blue trend is the power generated by XoSoft. For what concerns the power generated by XoSoft on the hip joint (Fig. 10(a)), it presents negative values between $20 \%$ and $50 \%$ of gait. Thus, power is subtracted to the user, and it characterizes the storing phase of the actuator. Whereas, the positive part of the power trend is the releasing phase of the actuator. This positive energy corresponds to the assistance given to the user by augmenting their power. In fact, the blue trend represents the right hip power of the user wearing XoSoft, where, in the region between 50\% and $70 \%$ of gait, it is augmented with respect to the INX resulting similar to the left unaffected hip trend. In the gait region between $30 \%$ and 50\%, the right hip power has a lower value with respect to the INX condition (red trend), thus showing the need for the user to expend more energy before receiving the assistance.

The effect on the knee is slightly less evident. The main effect is the augmentation of power as soon as the XoSoft power (light blue trend) rises giving assistance (positive value) within the range of gait between $50 \%$ and $65 \%$. Thus, the blue trend is pushed up by XoSoft in correspondence of the toe off ( $60 \%$ of gait). Therefore, the resulting behavior of right knee power wearing XoSoft is miming the left unaffected knee power within the range $40-100 \%$ of the gait. Moreover, the negative spike of right knee INX power, present at around $65 \%$ of gait, has been reduced by using XoSoft.

Figure 11 presents average assistance in terms of $\Lambda$ (expressed in Eq. (6)) under all the control conditions $\left(P A, Q P A_{A}\right.$, and $Q P A_{B}$ combined with $E B_{A}$ and $\left.E B_{B}\right)$. As previously mentioned, $\Lambda=1$ means that the prototype is not providing any assistance, while $\Lambda=0$ represents $100 \%$ of assistance. The assistance in terms of $\Lambda$ for hip and knee joint is summarized in Table $V$ for each condition of control applied. The average assistance, considering six tested conditions, for hip is $10.9 \% \pm 2.2 \%$ and for knee is $9.3 \% \pm 3.5 \%$.

Table $\mathrm{V}$ also reports the storing and releasing power that actuation modules are sharing with hip and knee articulations. These parameters are directly related to the amount of contribution XoSoft generates on the resultant user's joint power. The percentage of storing is calculated as ratio of XoSoft power over the user's joint power within the gait section where the exoskeleton power is negative. A negative XoSoft power is correlated with the fatigue experienced by the user during storing phase. This range of gait is further distinguished in relation with the user's power as (--), if the user's power is also negative, or $(-+)$, if positive. Therefore, the storing percentage (--) results are concordant with the eccentric muscle contraction. Thus, it contributes with the energy dissipation related to the task. Storing percentage, labeled as $(-+)$, describes the amount of time when the device is dissipating energy while the user is generating energy instead. Thus, the device is countering generation of power related to the user's task. It is definitely preferable if XoSoft and user's power are concordant, otherwise the user is generating energy while the device is working against. Moreover, a small value of both two storing ratios mean that the user does not feel fatigue (the exoskeleton results being transparent), and the device works in a physiological manner with the user's motion. 

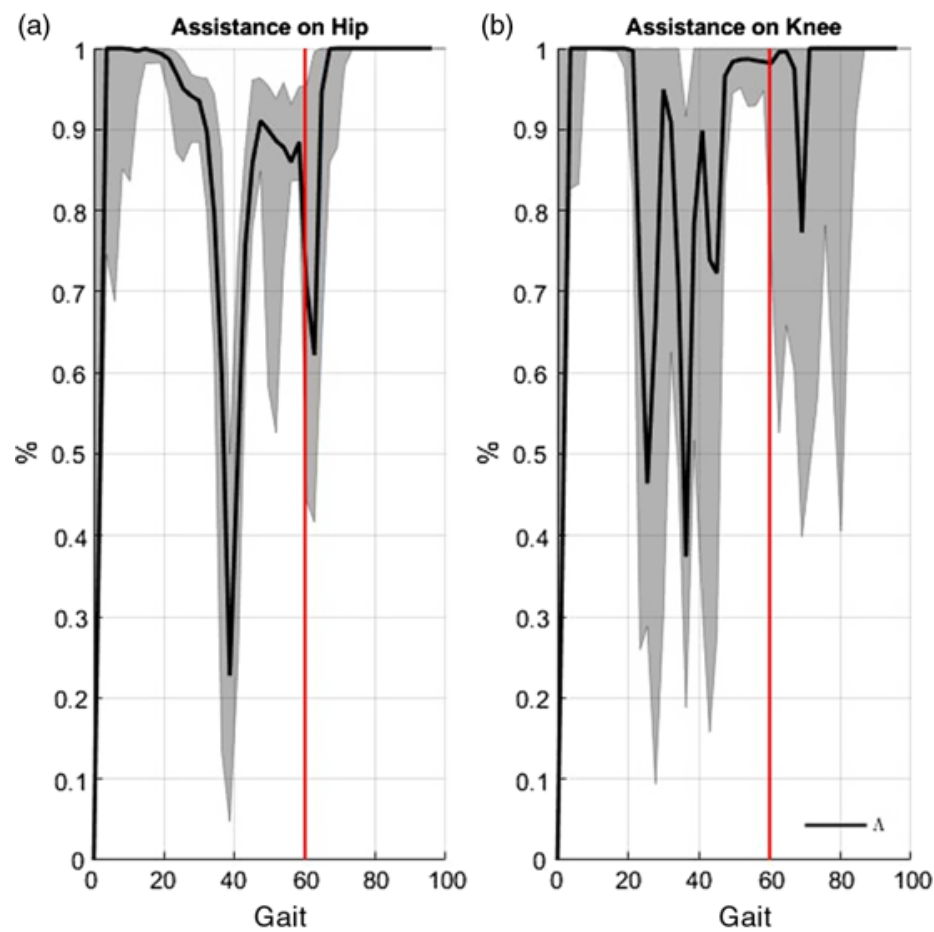

Fig. 11. Comparison of hip (a) and knee (b) assistance for the right leg with XoSoft under all the control conditions $\left(P A, Q P A_{A}\right.$, and $\left.Q P A_{B}\right)$ and different $E B s$. The vertical lines represent the instant when toe off occurs for right gait.

Similarly, the releasing percentage is also measured as ratio between the power generated by XoSoft and the joint power within the gait region where the actuator's power is positive. Within the releasing power, a further distinction is evaluated between $(++)$ and $(+-)$. The releasing power ratio $(++)$ is when both powers are concordant, while the $(+-)$ power ratio is when the user's power is negative. The $(++)$ releasing power ratio is correlated with the net assistance transmitted to the user, and it is correlated with energy generation during concentric muscle contraction. The $(+-)$ power represents a lack of concordance between the energy released by the device and user motion, which correlates with eccentric muscle contraction. Therefore, a high value of releasing $(++)$, associated with a concentric muscular contraction, will generate assistance to user articulation, because the work done by XoSoft is parallel to the joint motion. In contrast, the releasing $(+-)$ is associated with an eccentric muscular contraction; thus the system is working contrary to the muscle activation, performing as energy dissipater. One of the outputs researched by the knee actuation is behaving as dissipative element to reduce the hyper-extension during the last part of the swing phase. This is possible only if control strategy $P A$ is exploited, but the angle trend does not change with respect to INX.

In conclusion, if considering the percentage of releasing $(++)$, the hip module generates a $12.3 \% \pm 2.8 \%$ and the knee module generates a $7.7 \% \pm 3.3 \%$ of net assistance.

\subsection{Results: foot clearance consideration}

Figure 12 presents the kinematic evaluation of the right and left lower limbs. The foot clearance variation of right foot with respect to the INX condition is evaluated in the middle between mid-swing and heel strike. The results, summarized in Table VI, show the difference in foot clearance of right foot under the six studied conditions $\left(P A, Q P A_{A}\right.$, and $Q P A_{B}$ combined with $E B_{A}$ and $\left.E B_{B}\right)$ with respect to the right foot $I N X$. The second table column displays the foot clearance of the right versus left foot during the same control conditions. It is evident that XoSoft improved foot clearance in any control condition with an average of $5.4 \pm 0.45 \mathrm{~cm}$ in case of using $E B_{A}$ and $3.5 \pm 1.5 \mathrm{~cm}$ for $E B_{B}$. This result clearly shows that foot clearance performs better with $E B_{A}$ if considering the comparison with the right side under INX condition. The same result is confirmed by the comparison of the foot clearance with respect to the left foot. The right foot clearance is improved with respect to the pathological condition but still lower than the unaffected user's left leg with an average of $-1.5 \pm 0.55 \mathrm{~cm}$ for $E B_{A}$ and $-3.5 \pm 1.5 \mathrm{~cm}$ for $E B_{B}$. 
Table VI. Foot clearance value under the following conditions $P A, Q P A_{A}, Q P A_{B}$ and $E B_{A}, E B_{B}$ respect to the right $I N X$.

\begin{tabular}{lcc}
\hline Condition & $\begin{array}{c}\Delta \text { Foot clearance with } \\
\text { respect to right } \boldsymbol{I N X}(\mathbf{c m})\end{array}$ & $\begin{array}{c}\boldsymbol{\Delta} \text { Foot clearance with } \\
\text { respect to left side }(\mathbf{c m})\end{array}$ \\
\hline$P A$ and $E B_{A}$ & 5.8 & -1.0 \\
$Q P A_{A}$ and $E B_{A}$ & 5.4 & -2.1 \\
$Q P A_{B}$ and $E B_{A}$ & 4.9 & -1.5 \\
$P A$ and $E B_{B}$ & 5.2 & -1.8 \\
$Q P A_{A}$ and $E B_{B}$ & 2.6 & -4.2 \\
$Q P A_{B}$ and $E B_{B}$ & 2.7 & -4.7 \\
\hline
\end{tabular}

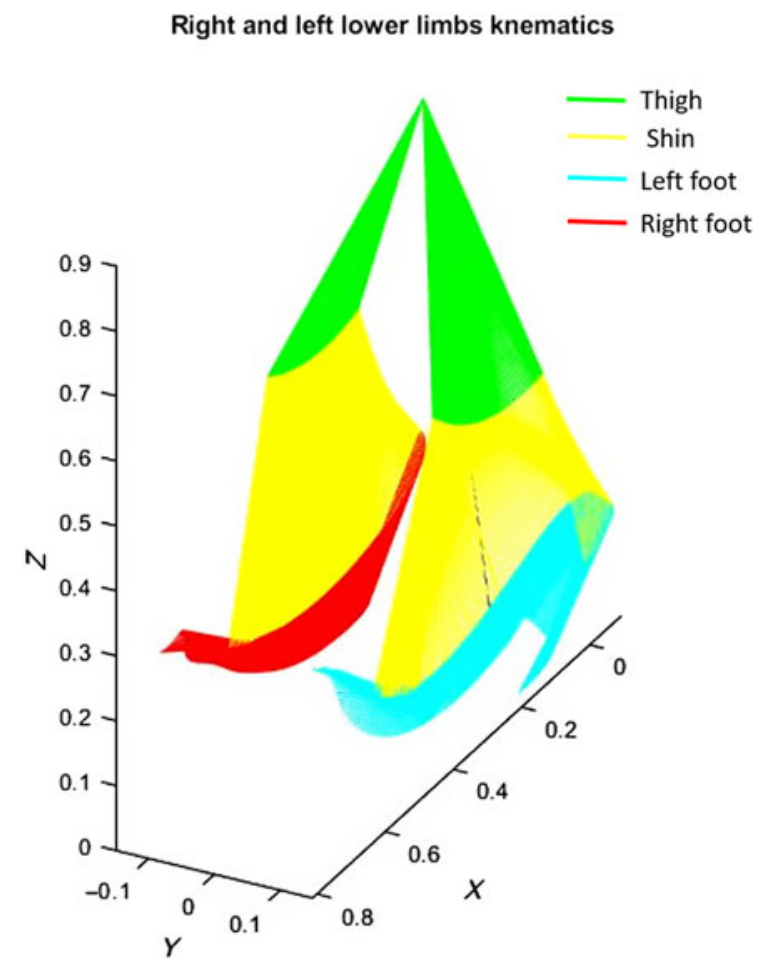

Fig. 12. Direct kinematics of lower limbs during gait.

\subsection{Results: best configuration}

Best actuator design and control strategy for hip and knee based on the above results of power ratio and foot clearance (summarized in Tables V and VI) are presented and analyzed in this section. From Table $\mathrm{V}$, the selected hip configuration is the $Q P A_{B}$ with $E B_{A}$ because the storing power rate $(-+)$ is lower than others, and the releasing power rate $(++)$ is $12.7 \%$. Hip angle trend, power, and $\Lambda$ are plotted in Fig. 13(a)-(c). Figure 13(a) shows how quickly the hip angle reaches approximately $30^{\circ}$ at the beginning of the swing due to the power released. This behavior is similar in terms of angular rate of variation to the healthy user's hip angle. From the power point of view (Fig. 13(b)), the right hip can generate more power than the INX configuration within 50-60\% of gait. Also, the system dissipates more power with respect to the affected right hip, but it remains within the healthy range of power. $\Lambda$ is displayed in Fig. 13(c), and its main effect is between $50 \%$ and $65 \%$, thus strongly effecting the power trend.

The knee best configuration, from Table $\mathrm{V}$, results being $Q P A_{B}$ with $E B_{A}$, since both the storing powers are very low (about 3\%) and the $(++)$ releasing power rate is $7 \%$. Figure 13(d) shows the knee angle and the angle peak at mid-swing is about the same of left knee. The knee angle during stance does not present a healthy range of motion instead. Figure 13(e) displays the power trend. The power at the initial swing is increased reaching the left knee power. $\Lambda$, displayed in Fig. 13(f), 
(a)

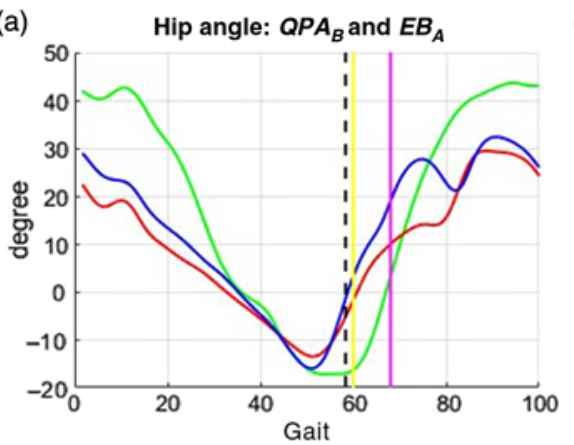

(b)

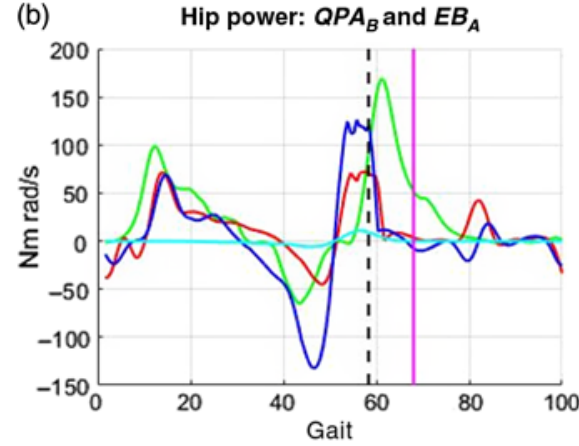

(c)

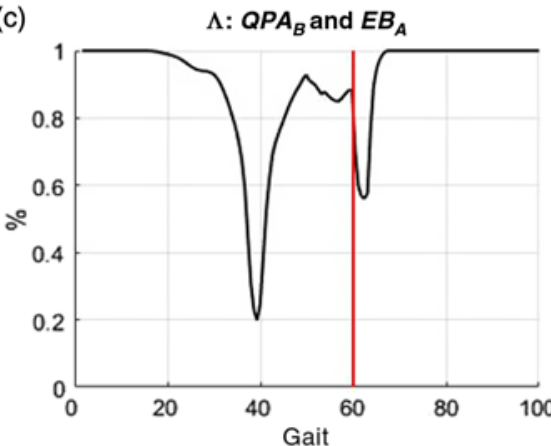

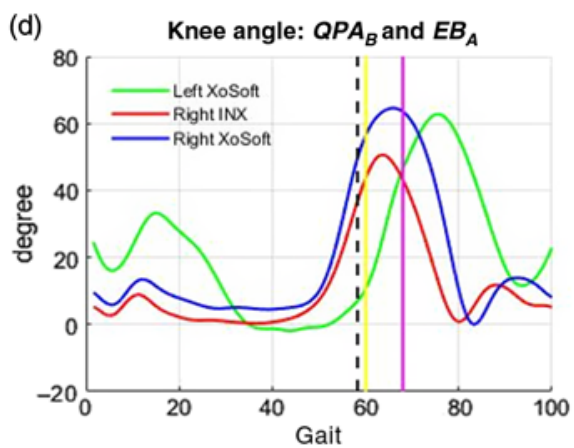

(e)

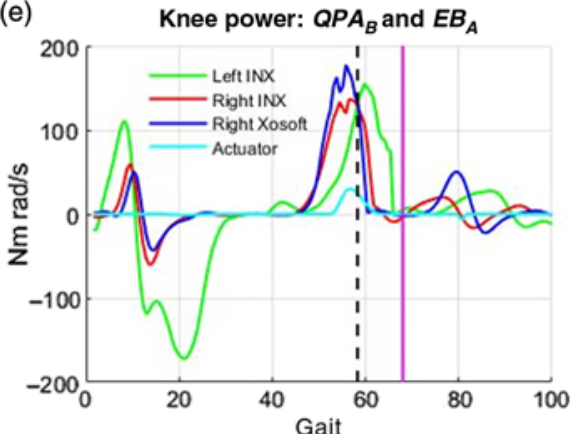

(f)

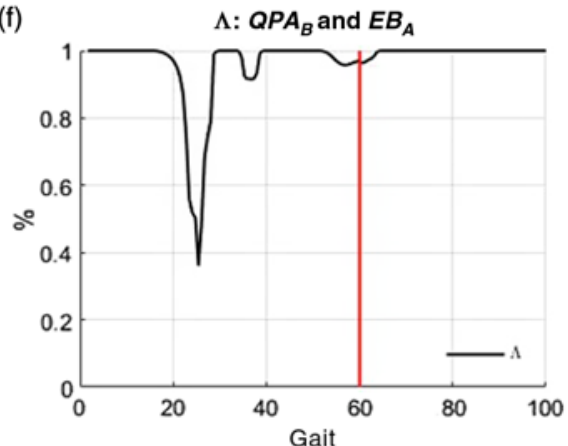

Fig. 13. Comparison of hip (a)-(c) and knee (d)-(f) joint angle, power, and assistance for right and left legs with XoSoft and $I N X$ under the unique control condition $Q P A_{B}$ and $E B_{A}$. The vertical lines represent the instant when toe off occurs.

describes a good amount of storing and releasing phase about $30 \%$ and $55 \%$ of gait, respectively. However, since the hyper-extension is not reduced, this control strategy seems being not effective to address this problem.

\section{Conclusions}

This paper presents a novel design and experimental validation of a modular soft exoskeleton for lower limb assistance (XoSoft EU project). The work presented is a modular actuation unit concept based on quasi-passive mechanical elements used to transfer energy between different gait phases, thus reducing needed power to accomplish daily tasks with a total weight of $4.9 \mathrm{~kg}$.

The exoskeleton was assessed through human experimental section, conducted as described in Section 3, where three control strategies $\left(P A, Q P A_{A}\right.$, and $\left.Q P A_{B}\right)$ and two different $E B s\left(E B_{A}\right.$ and $E B_{B}$ ) were evaluated and compared.

The energy analysis of the human-exoskeleton interaction in relation to task-based biological power generation has been useful to select the best control configuration. This analysis approach could be also beneficial to exoskeleton design procedure to accomplish different tasks.

The results reveal a net assistance offered by the $E B_{A}$, considering the average of all the control strategies $\left(P A, Q P A_{A}\right.$, and $\left.Q P A_{B}\right)$ and measured as releasing ratio, of $13 \%$ for the hip actuation and $7 \%$ for knee. This $E B$ performs better than $E B_{B}$, thus conducting the actuation design to the proper $E B$ 
selection. Moreover, the XoSoft can improve the mobility and power range of the affected leg joints as displayed in Figs. 8 and 10. The foot clearance (Table VI) is also affected by the exoskeleton, showing an increase of about $5 \mathrm{~cm}$ if $E B_{A}$ is applied. The control strategy $\left(Q P A_{B}\right)$ adopted for the hip and knee configuration seems effective for the postural and power reduction consideration. Nevertheless, the knee control strategy is not completely effective since hyper-extension is not reduced. Further, more complex actuation strategy will be implemented, such as a second engagement-disengagement phase during swing. Note that training phase was not conducted before the experimental evaluation; therefore, the data was not effected by a learning phase.

By comparing the achievements of similar soft exoskeletons presented in the literature, it is remarkable that active systems such as Awad et al. ${ }^{12}$ and Jin et al. ${ }^{13}$ are able to generate an assistance of $12 \%$ and $7 \%$, respectively. The assistance evaluated in this study for the Beta 1 prototype with a $Q P A$ is $10.9 \%$ and $9.3 \%$ for hip and knee, respectively, as determined by the $\Lambda$ value.

While a statistically relevant study was outside the scope of this preliminary study, these results of this modular wearable device encourage for further investigations on multiple joint actuation segments and more statistical objectivity.

As previously mentioned, further improvements to the exoskeleton will be undertaken to develop multiple joint actuations based on soft technology, specific soft sensing ${ }^{25}$ to measure user's angular joint and enable a more accurate control strategy.

Also, it is significant to emphasize the main role of the users since the exoskeleton is interacting strongly with them. In fact, the exoskeleton with $Q P A$ needs the input force by the wearers to release energy back to them again. Therefore, the design of a framework to address modeling of the interaction between human and exoskeleton could serve as an important tool to better understand complex systems such as wearable devices.

Future works involve determining how XoSoft affects metabolic cost.

\section{Acknowledgments}

This work has received funding from the European Union's Horizon 2020 framework programme for research and innovation under grant agreement no. 688175.

\section{References}

1. Available at http://ec.europa.eu/eurostat/statistics-explained/index.php/Population_structure_and_ageing/.

2. K. Lakshminarayan, A. K. Berger, C. C. Fuller, D. R. Jacobs, D. C. Anderson, L. M. Steffen, A. Sillah and R. V. Luepker, "Trends in 10-year survival of patients with stroke hospitalized between 1980 and 2000: The Minnesota stroke survey," Stroke 45(9), 2575-2581 (2014).

3. T. Yan, M. Cempini, C. M. Oddo and N. Vitiello, "Review of assistive strategies in powered lower-limb orthoses and exoskeletons," Rob. Auton. Syst. 64, 120-136 (2015).

4. Y. Sankai, "HAL: Hybrid Assistive Limb Based on Cybernics," In: Robotics Research (Springer, Berlin, Heidelberg, 2010) pp. 25-34.

5. S.-H. Hyon, J. Morimoto, T. Matsubara, T. Noda and M. Kawato, "XoR: Hybrid Drive Exoskeleton Robot that can Balance," 2011 IEEE/RSJ International Conference on Intelligent Robots and Systems (IROS), San Francisco, CA, USA (IEEE, 2011) pp. 3975-3981.

6. M. Jun, N. Tomoyuki and H. Sang-Ho, "Extraction of Latent Kinematic Relationships Between Human Users and Assistive Robots," 2012 IEEE International Conference on Robotics and Automation (ICRA), St. Paul, Minnesota, USA (2012) pp. 3090-3915.

7. T.-J. Yeh, M.-J. Wu, T.-J. Lu, F.-K. Wu and C.-R. Huang, "Control of McKibben pneumatic muscles for a power-assist, lower-limb orthosis," Mechatronics 20(6), 686-697 (2010).

8. T. Nakamura, K. Saito, Z. Wang and K. Kosuge, "Realizing Model-Based Wearable Antigravity Muscles Support with Dynamics Terms," 2005 IEEE/RSJ International Conference on Intelligent Robots and Systems, 2005, IROS 2005, Edmonton, Alberta, Canada (IEEE, 2005) pp. 2694-2699.

9. T. Nakamura, K. Saito and K. Kosuge, "Control of Wearable Walking Support System Based on HumanModel and GRF," Proceedings of the 2005 IEEE International Conference on Robotics and Automation, 2005, ICRA 2005, Barcelona, Spain (IEEE, 2005) pp. 4394-4399.

10. T. Ikehara, K. Nagamura, T. Ushida, E. Tanaka, S. Saegusa, S. Kojima and L. Yuge, "Development of Closed-Fitting-Type Walking Assistance Device for Legs and Evaluation of Muscle Activity," 2011 IEEE International Conference on Rehabilitation Robotics (ICORR), Zurich, Switzerland (IEEE, 2011) pp. 1-7.

11. K. Kong and D. Jeon, "Design and control of an exoskeleton for the elderly and patients," IEEE/ASME Trans. Mechatron. 11(4), 428-432 (2006).

12. L. N. Awad, J. Bae, K. O’Donnell, S. M. De Rossi, K. Hendron, L. H. Sloot, P. Kudzia, S. Allen, K. G. Holt, T. D. Ellis, and C. J. Walsh, "A soft robotic exosuit improves walking in patients after stroke," Sci. Trans. Med. 9(400), eaai9084 (2017). 
13. S. Jin, N. Iwamoto, K. Hashimoto and M. Yamamoto, "Experimental evaluation of energy efficiency for a soft wearable robotic suit," IEEE Trans. Neural Syst. Rehabil. Eng. 25(8), 1192-1201 (2017).

14. K. Schmidt, J. E. Duarte, M. Grimmer, A. Sancho-Puchades, H. Wei, C. S. Easthope and R. Riener, "The myosuit: Bi-articular anti-gravity exosuit that reduces hip extensor activity in sitting transfers," Front. Neurorob. 11, 57 (2017).

15. J. Ortiz, E. Rocon, V. Power, A. de Eyto, L. O’Sullivan, M. Wirz, C. Bauer, S. Schülein, K. S. Stadler, B. Mazzolai, and W. B. Teeuw, "XoSoft-A Vision for a Soft Modular Lower Limb Exoskeleton," In: Wearable Robotics: Challenges and Trends (Springer, Cham, 2017) pp. 83-88.

16. V. Power, L. O'Sullivan, A. de Eyto, S. Schülein, C. Nikamp, C. Bauer, J. Mueller and J. Ortiz, "Exploring User Requirements for a Lower Body Soft Exoskeleton to Assist Mobility," Proceedings of the 9th ACM International Conference on PErvasive Technologies Related to Assistive Environments, Corfu, Greece (ACM, 2016) p. 69.

17. J. Ortiz, T. Poliero, G. Cairoli, E. Graf and D. G. Caldwell, "Energy efficiency analysis and design optimization of an actuation system in a soft modular lower limb exoskeleton," IEEE Rob. Autom. Lett. 3(1), 484-491 (2018).

18. T. Poliero, C. Di Natali, M. Sposito, J. Ortiz, E. Graf, C. Pauli, E. Bottenberg, A. de Eyto and D. G. Caldwell, "Soft Wearable Device for Lower Limb Assistance: Assessment of an Optimized Energy Efficient Actuation Prototype," IEEE-RAS International Conference on Soft Robotics (RoboSoft), Livorno, Italy (IEEE, 2018) pp. 559-564.

19. K. Endo, D. Paluska and H. Herr, "A Quasi-passive Model of Human Leg Function in Level-Ground Walking," 2006 IEEE/RSJ International Conference on Intelligent Robots and Systems, Beijing, China (IEEE, 2006) pp. 4935-4939.

20. D. A. Winter, Biomechanics and Motor Control of Human Gait: Normal, Elderly and Pathological (University of Waterloo Press, Ontario, Canada, 1991).

21. J. Mecheels and K. H. Umbach, "The Psychrometric Range of Clothing Systems," In: Clothing Comfort (Ann Arbor Science, Michigan, 1977) pp. 133-151.

22. L. A. Mateos, J. Ortiz, S. Toxiri, J. Fernández, J. Masood and D. G. Caldwell, "Exoshoe: A Sensory System to Measure Foot Pressure in Industrial Exoskeleton," 2016 6th IEEE International Conference on Biomedical Robotics and Biomechatronics (BioRob), Singapore (IEEE, 2016), pp. 99-105.

23. I. Söderkvist and P.-Å. Wedin, "Determining the movements of the skeleton using well-configured markers," J. Biomech. 26(12), 1473-1477 (1993).

24. E. S. Grood and W. J. Suntay, "A joint coordinate system for the clinical description of three-dimensional motions: Application to the knee," J. Biomech. Eng. 105(2), 136-144 (1983).

25. M. Totaro, T. Poliero, A. Mondini, C. Lucarotti, G. Cairoli, J. Ortiz and L. Beccai, "Soft smart garments for lower limb joint position analysis," Sensors 17(10), 2314 (2017).

26. R. List, T. Gülay, M. Stoop and S. Lorenzetti, "Kinematics of the trunk and the lower extremities during restricted and unrestricted squats," J. Strength Conditioning Res. 27(6), 1529-1538 (2013).

27. G. Wu, S. Siegler, P. Allard, C. Kirtley, A. Leardini, D. Rosenbaum, M. Whittle, D. D. D'Lima, L. Cristofolini, H. Witte, and O. Schmid, "ISB recommendation on definitions of joint coordinate system of various joints for the reporting of human joint motion-part I: Ankle, hip, and spine," J. Biomechan. 35(4), 543-548 (2002).

28. G. Wu and P. R. Cavanagh, "ISB recommendations for standardization in the reporting of kinematic data," J. Biomechan. 28(10), 1257-1261 (1995).

29. D. A. Winter, Biomechanics and Motor Control of Human Movement (John Wiley \& Sons, NJ, USA, 2009).

\section{Appendix: Kinematics and Dynamics Calculations}

\section{A.1. Definition of virtual markers and segment coordinate systems}

Based on the markers placed on anatomical landmarks (Fig. A.1), the following virtual markers are calculated:

$$
\begin{array}{cc}
\text { Left side } & \text { Right side } \\
L I M=(L A L M+L A M M) / 2 & R I M=(R A L M+R A M M) / 2 \\
L I C=(L K L E+L K M E) / 2 & R I C=(R K L E+R K M E) / 2 \\
& \\
S A C R=(L P S I+R P S I) / 2
\end{array}
$$

Functional calibration tasks were executed to determine the knee axis and the hip joint center to decrease the effect of variability in marker placement and achieve higher accuracies. For the knee, three repetitions of unloaded knee flexion during upright standing were performed. For the hip, three repetitions of hip circumduction were performed. A helical axis approach was used to determine the knee axis and joint center (see List et al. ${ }^{26}$ for more details). 


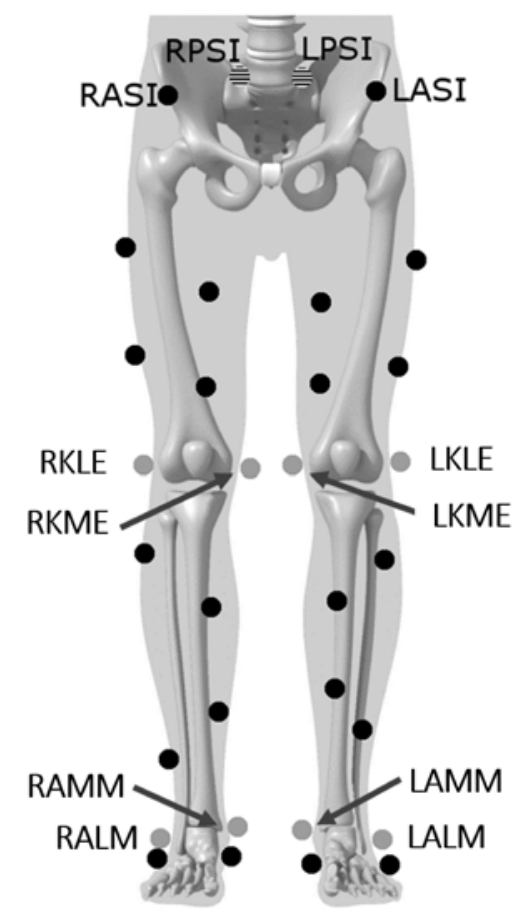

Fig. A.1. Physical markers placed on the lower extremities and pelvis. Black: tracking markers that are used to track the segment during dynamic trials (except markers on pelvis which are used as tracking and segment coordinate system defining markers). Gray: markers placed on anatomical landmarks used to define the segment coordinate systems. Black-white: placed on the posterior aspect of the body.

The right-handed coordinate systems for each segment are defined individually based on virtual and physical markers. The segment coordinate system definition depends on the joint for which the angle is calculated. All definition follow the recommendations of $\mathrm{Wu}$ et al. ${ }^{27}$

Ankle joint: Rear-foot coordinate system definition

$$
\begin{array}{cc}
\text { Left side } & \text { Right side } \\
L R F_{C S \text { origin }}=L I M & R R F_{C S o r i g i n}=R I M \\
L R F_{C S y}=L I M-L I C & R R F_{C S y}=R I M-R I C \\
t e m p_{1}=L A M M-L A L M & \text { temp }_{2}=R A L M-R A M M \\
L R F_{C S x}=L R F_{C S y} \times t e m p_{1} & R R F_{C S x}=R R F_{C S y} \times t e m p_{2} \\
L R F_{C S z}=L R F_{C S x} \times L R F_{C S y} & R R F_{C S z}=R R F_{C S x} \times R R F_{C S y}
\end{array}
$$

Ankle joint: Shank coordinate system definition

$$
\begin{gathered}
\text { Left side } \\
L S H A_{C S o r i g i n}=L I M \\
L S H A_{C S z}=L A M M-L A L M \\
L S H A_{C S y}=L S H A_{C S z} \times L R F_{C S x} \\
L S H A_{C S x}=L S H A_{C S y} \times L S H A_{C S z}
\end{gathered}
$$

$$
\begin{gathered}
\text { Right side } \\
R S H A_{C S o r i g i n}=R I M \\
R S H A_{C S z}=R A L M-R A M M \\
R S H A_{C S y}=R S H A_{C S z} \times R R F_{C S x} \\
R S H A_{C S x}=R S H A_{C S y} \times R S H A_{C S z}
\end{gathered}
$$

Knee joint: Shank coordinate system definition

Left side

$$
\begin{gathered}
L_{S S H} K_{C S o r i g i n}=L I C \\
L S H K_{C S z}=L I C-L I M \\
L S H K_{C S y}=L S H K_{C S z} \times L K A \\
L S H K_{C S x}=L S H K_{C S y} \times L S H K_{C S z}
\end{gathered}
$$

Right side

$$
\begin{gathered}
R_{S H K_{C S o r i g i n}}=R I C \\
R S H K_{C S z}=R I C-R I M \\
R S H K_{C S y}=R S H K_{C S z} \times L K A \\
R S H K_{C S x}=R S H K_{C S y} \times R_{S H K} \text { CSz }
\end{gathered}
$$


Knee joint: Thigh coordinate system definition

$$
\begin{aligned}
& \text { Left side } \\
& \text { LTHK }_{\text {CSorigin }}=L I C \\
& L T H K_{C S x}=L A M M-L A L M \\
& \text { temp }_{3}=L H J C-L I C \\
& L_{T H} K_{C S y}=t e m p_{3} \times L I C \\
& L T H K_{C S z}=L T H K_{C S x} \times L T H K_{C S y} \\
& R T H K_{\text {CSorigin }}=R I C \\
& R_{T H K} K_{C S z}=R A L M-R A M M \\
& \text { temp }_{4}=\text { RHJC }- \text { RIC } \\
& R T H K_{C S y}=\text { temp }_{4} \times R I C \\
& R T H K_{C S z}=R T H K_{C S x} \times R T H K_{C S y}
\end{aligned}
$$

$\mathrm{L} / \mathrm{RKA}$ is the vector representation of the left/right knee joint rotational axis, whereas L/RHJC is the left/right hip joint center.

Hip joint: Thigh coordinate system definition

$$
\begin{aligned}
& \text { Left side } \\
& \text { LTHH }_{\text {CSorigin }}=\text { LHJC } \\
& L T H H_{C S y}=L H J C-L I C \\
& \text { temp }_{5}=\mathrm{LTHH}_{C S y} \times L K A \\
& \mathrm{LTHH}_{\mathrm{CSz}}=\operatorname{temp}_{5} \times \mathrm{LTHH}_{\mathrm{CSy}} \\
& L T H H_{C S x}=L T H H_{C S y} \times L T H H_{C S z} \\
& \text { Right side } \\
& R_{T H} H_{C S o r i g i n}=R H J C \\
& R T H H_{C S y}=R H J C-R I C \\
& \text { temp }_{6}=\mathrm{RTHH}_{\mathrm{CSy}} \times \mathrm{RKA} \\
& R T H H_{C S z}=\operatorname{temp}_{6} \times \mathrm{RTHH}_{\mathrm{CSy}} \\
& R T H H_{C S x}=R T H H_{C S y} \times R T H H_{C S z}
\end{aligned}
$$

Knee joint: Pelvis coordinate system definition

$$
\begin{gathered}
\text { Left side } \\
\text { LPEH }_{C S \text { origin }}=L H J C \\
L P E H_{C S z}=R A S I-L A S I \\
\text { temp }_{7}=(R A S I-L A S I) / 2-S A C R \\
\text { temp }_{8}=\text { LPEH }_{C S z} \times \text { temp }_{7} \\
\text { LPEH }_{C S x}=\text { temp }_{8} \times \text { LPEH }_{C S z} \\
\text { LPEH }_{C S y}=\text { LPEH }_{C S z} \times \text { LPEH }_{C S x}
\end{gathered}
$$

$$
\begin{gathered}
\text { Right side } \\
R P E H_{C S o r i g i n}=R H J C \\
R P E H_{C S z}=R A S I-R A S I \\
\text { temp }_{9}=(R A S I-L A S I) / 2-S A C R \\
\text { temp }_{10}=R P E H_{C S z} \times \text { temp }_{9} \\
R P E H_{C S x}=\text { temp }_{10} \times \text { RPEH }_{C S z} \\
\text { RPEH }_{C S y}=\text { RPEH }_{C S z} \times \text { RPEH }_{C S x}
\end{gathered}
$$

\section{A.2. Kinematics and inverse dynamics}

The joint angles are calculated according to joint coordinate systems which is based on an axis fixed to the proximal segment $\left(e_{1}\right)$, an axis fixed to the distal segment $\left(e_{3}\right)$, and an axis perpendicular to the other two axes $\left(e_{2}=e_{1} \times e_{3}\right){ }^{28}$ Based on standardization joint rotations are defined as follows:

- Rotation around $e_{1}$ : flexion (ankle: dorsiflexion).

- Rotation around $e_{2}$ : adduction (ankle: inversion).

- Rotation around $e_{3}$ : internal rotation.

Ankle (calculation according to Wu et al. ${ }^{27}$ )

$$
e_{1}=R / L S H A_{C S z} e_{3}=R / L S R F_{C S y}
$$

Knee (calculation according to Grood et al. ${ }^{24}$ )

$$
e_{1}=R / L_{T H K} K_{C S x}=R / L S H K_{C S z}
$$

Hip (calculation according to Wu et al. ${ }^{27}$ )

$$
e_{1}=R / L_{C P E} H_{C S} e_{3}=R / L T H H_{C S y}
$$

The calculations of internal forces and torques follow the laws of motion and general principles of biomechanics. A detailed explanation of the calculation can be found in textbooks. ${ }^{29}$ The following assumptions are made during calculations:

- Each segment is represented by a point mass located at the center of mass (which is at a fixed location) of the segment.

- All joints are assumed as hinge.

- For each segment, the mass moment of inertia is constant.

- The length of each segment does not change during movement. 